\title{
E1 sistema "compuesto" de manejo de residuos en Lima: ¿cómo sacar provecho de las prácticas en las ciudades en desarrollo? ${ }^{1}$
}

The "composite" waste management system en Lima: how to profit from practices in developing cities

Mathieu Durand ${ }^{2}$

\section{Resumen}

Las autoridades de las ciudades en desarrollo tienen por objetivo manejar sus residuos sólidos según modelos internacionales, que dan prioridad a la eliminación de los residuos sobre su valorización y a la red de actores formales sobre los informales. Ahora bien, la realidad socioeconómica no permite alcanzar estos objetivos. Las ciudades no tienen los recursos financieros o humanos para colectar y eliminar todos sus residuos, entonces se organizan redes informales para remplazar a las autoridades.

Existen muchos ejemplos que muestran que un acercamiento es posible entre los actores informales y las autoridades públicas. Se trata de mejorar la calidad del servicio, realizando un reciclaje más efectivo, atrayendo a los recicladores informales hacia una regularización de sus actividades y un mejoramiento de sus condiciones de trabajo. Este proceso es largo y complejo porque obliga a las autoridades a ubicarse al margen de la ley. Mientras tanto, permite un mejoramiento muy progresivo pero real del sistema de manejo de residuos sólidos. En este artículo se busca detallar el funcionamiento de tres sistemas de gestión de residuos en la ciudad de Lima: la gestión "pública", la "autogestión" y la gestión "compartida". De este modo, se notará que un sistema "compuesto" se organiza, buscando sacar provecho de una situación local que no permite ofrecer la mejor solución técnica, ambiental o sanitaria. Sin embargo, este sistema compuesto sí permite mejorar de manera progresiva la situación de las ciudades en desarrollo.

1 Este texto es el resultado de una investigación desarrollada en el marco de una tesis de doctorado en la Universidad de Rennes 2 (Francia), bajo la dirección de Vincent Goueset (Universidad de Rennes 2) y Robert D’Ercole (Instituto de Investigación RD)..

2 Doctor en Geografía. Filiación Institucional: Profesor de Ordenamiento Territorial en la Universidad du Maine (Francia), miembro del centro de investigación ESO (Espacio y Sociedad) del CNRS (Centro Nacional de la Investigación Científica). País: Francia. Correo electrónico: Mathieu.durand@univ-lemans.fr 
Palabras clave: Gestión ambiental, Lima, Perú, tratamiento de desechos.

\section{Résumé}

Les gestionnaires des villes en développement ont pour objectif de gérer leurs déchets en fonction de standards de qualité internationaux. Ceux-ci privilégient l'élimination des déchets sur leur valorisation, le réseau d'acteurs formel sur l'informel. Or la réalité socio-économique ne permet pas d'atteindre ces objectifs. Les villes n'ont en effet ni les moyens financiers, ni les moyens humains de collecter et d'éliminer tous leurs déchets. Par conséquent, des réseaux informels se mettent en place afin de palier les déficiences des pouvoirs publics (et des entreprises prestataires de services).

Or de plus en plus de voix se font entendre pour opérer un rapprochement entre les acteurs informels et les pouvoirs publics. II s'agit d'améliorer la qualité du service, notamment en opérant un recyclage plus poussé des déchets, tout en attirant les recycleurs informels vers une régularisation de leur activité et vers une amélioration de leurs conditions de travail. Ce processus est long et complexe puisqu'il oblige les pouvoirs publics à être à la marge de la légalité. II permet cependant une amélioration très progressive mais réelle du système de gestion des déchets. Cette communication cherchera à détailler le fonctionnement des trois systèmes de gestion des déchets : la gestion «publique», «l'autogestion» et la gestion «partagée» dans la ville de Lima. Nous verrons ainsi qu'un système de gestion "composite» se met alors en place, cherchant à tirer profit d'une situation locale ne permettant pas toujours d'offrir la meilleure solution technique, environnementale ou sanitaire, mais permettant une amélioration progressive de la situation.

Mots Clés: Gestion de l'environnement, Lima, Perú, Traitement des déchets.

\section{Abstract}

The objective of development cities administrators is to manage their waste according to international quality standards. They give priority to the elimination of urban waste above valorization,formal actors above informal. But the socioeconomic reality does not allow reaching these objectives. Cities have indeed neither the financial means, nor average human beings to collect and to eliminate all their waste. Consequently, informal networks are set up to manage the deficiencies of public authorities (and companies' service providers).

There are many examples which illustrate a link is possible between informal actors and the public authorities. It is a question of improving the quality of the service, in particular by operating a more effective recycling program, while attracting the informal recyclers towards a regularization of their activity 
and towards an improvement of their working conditions. This process is long and complex because it obliges public authorities to be outside of the law. It allows however a very progressive but real improvement of the waste management system. This communication will try to detail the functioning of three waste management systems: the "public management", the "selfmanagement" and the "shared management" in the city of Lima. We will notice that a "composite" management system is then set up, trying to benefit from a local situation that does not allow offering the best technical, environmental or sanitary solution, but allows a progressive improvement of the situation.

Keywords: Environmental management, Lima, Perú, Waste treatment, 


\section{Introducción}

Las autoridades de las ciudades de los países en desarrollo frecuentemente siguen los modelos publicitados por las ciudades de los países del Norte. El objetivo pasa a ser que su propia ciudad alcance ese "buen estado" de funcionamiento y que el conjunto de su población sea atendida por un servicio de calidad estándar. Esa calidad corresponde a una evacuación casi total de los residuos sólidos y de las aguas residuales. La evacuación se hace a la periferia de la ciudad hacia infraestructuras que permiten su tratamiento (valorización, eliminación o almacenamiento). Ahora bien, las ciudades de los países en desarrollo intentan alcanzar este resultado en un contexto completamente distinto, puesto que ellas generalmente carecen de los medios financieros necesarios. En Europa, la implementación de estas redes se benefició, desde mediados del siglo XIX, del sostén activo de los Estados (Barraqué, 1988). En cambio, la debilidad de los Estados y de las municipalidades de los países en desarrollo, hace imposible una intervención a escala tan grande.

En este contexto, las ciudades deben frecuentemente contentarse con luchar contra los actores informales y las prácticas ilegales, a fin de limitar los aspectos más negativos de la situación. Esas prácticas se implementan como un sistema paralelo al legal, en la medida en que las municipalidades no están en capacidad de asegurar un servicio de calidad al conjunto de sus ciudadanos. Se trata de sistemas informales que no respetan las normas de protección medioambiental y de protección del riesgo sanitario, ni toman en cuenta los criterios sociales. Ahora bien, la existencia de estos servicios informales, a pesar de todo, permite a la ciudad funcionar y a los ciudadanos tener acceso al agua potable, a la electricidad y a la evacuación de sus aguas residuales y sus residuos sólidos.

La hipótesis del presente texto es que las municipalidades de los países en desarrolo podrían concentrar sus esfuerzos en conseguir una mejor articulación entre los sistemas formales e informales, en lugar de gastar sus recursos financieros y humanos en perseguir a los informales. El objetivo, evidentemente, no es bajar la calidad del servicio. Al contrario, se trata de avanzar hacia un mejoramiento progresivo del sistema, beneficiando al conjunto de los habitantes, pero teniendo en cuenta las circunstancias locales, sin pretender ir hacia un sistema utópico inaccesible. Esta reflexión se sustenta sobre unas experiencias que permiten demostrar que esta evolución es plausible. El caso de la gestión de los residuos sólidos en Lima (Perú) permitirá ilustrar esta propuesta.

Para realizar esta investigación, se ha hecho un trabajo de campo de cuatro años en la ciudad de Lima (2006-2010). Después, un trabajo más académico en Francia permitió analizar los datos recogidos, observar la bibliografía existente y evidenciar sistemas similares en otras ciudades latinoamericanas (Oliveira,2008 ${ }^{3}$,

3 Este texto se refiere a la experiencia de Curitiba (Brasil), ciudad innovadora en cuanto a la gestión social de los residuos. 
Carré, 2010, Fernández, 2012) ${ }^{4}$. Algunas investigaciones sobre la ciudades africanas también enseñan lógicas similares, hasta si el contexto urbano y socioeconómico es muy distinto de América Latina (Le Bris, 2002; Tchuikoua, 2010).

\section{Inspirarse en el modelo mundial de gestión de residuos: el sistema de "gestión pública"}

Antes de visualizar la cohabitación -y la evolución-de los diferentes sistemas de gestión de residuos en las ciudades en desarrollo, es necesario detallar el funcionamiento de cada uno de ellos. La especificidad de estas ciudades es que en el seno de la misma aglomeración urbana, encontramos tres sistemas de gestión de residuos sólidos, complementarios, articulados, pero con características distintas.

\subsection{Seguir el modelo de las ciudades del Norte}

El sistema de "gestión pública" es actualmente el más extendido en las ciudades latinoamericanas, al igual que en Europa o Norte América. Inspirado por los movimientos higienistas, empezó a aplicarse desde la segunda mitad del siglo XIX, con el objetivo de limitar con rapidez el impacto sanitario local de los residuos producidos por las poblaciones urbanas. Es decir, se trataba de evacuar

4 Estas dos últimas investigaciones son actualmente objeto de colaboraciones y de análisis comparados, en el marco de la red Sociedades Urbanas y Residuos (Sociétés Urbaines et Déchets) de la Universidad de Tours (Francia). los residuos al exterior de la ciudad (Barles 2005) y, en la medida de lo posible, eliminarlos.

En América Latina, tras una fase muy fuerte de crecimiento urbano posterior a la Segunda Guerra Mundial (Dureau, Goueset \& Mesclier, 2006), el principal objetivo de la gestión de los residuos sólidos fue evacuarlos, a fin de limitar los riesgos sanitarios incurridos por las poblaciones. Las municipalidades se enfocaron inicialmente en la etapa de la recolección, descuidando el tratamiento.

Así, en Lima, ciudad de 8,7 millones de habitantes ${ }^{5}$, casi el $90 \%{ }^{6}$ de la población tiene acceso a los servicios de recolección y evacuación de los residuos. De cara a las dificultades de gestión de los servicios urbanos, se ha dado prioridad a la cuestión cuantitativa (acceso al servicio del mayor número de personas) a expensas del aspecto cualitativo (efectividad del tratamiento). En un primer momento, las autoridades de la aglomeración urbana se han concentrado en la oferta de un servicio eficaz de colecta de los residuos domésticos.

En un segundo momento -siempre dentro del modelo de "gestión pública" eficaz-, las autoridades se han inclinado ante la necesidad de eliminar los residuos sólidos. Una vez que el asunto de la recolección está mejor implementado y una vez que el riesgo sanitario a corto plazo

5 Información del Censo del INEI (Instituto Nacional de Estadísticas e Informática) del 2007.

6 Estimaciones de las bases de datos municipales de las ciudades de Lima y Callao en 2008. 
- ligado a la permanencia de los desperdicios en medio de los habitantes- está controlado, es posible pasar a la etapa siguiente. Aquella se inició en el Perú, en el año 2000, con la promulgación de la Ley $\mathrm{N}^{\circ} 27314$ sobre los residuos sólidos. En ella se fijan de modo más preciso las responsabilidades de los diferentes actores en el nivel de cada etapa técnica de la gestión de los residuos, así como los procedimientos que deben ser utilizados.

La ley especifica que los residuos sólidos domésticos deben ser enviados a, y eliminados en, "rellenos sanitarios" (figura $\mathrm{N}^{\circ} 1$ ). Las municipalidades provinciales de la zona urbana de $\mathrm{Lima}^{7}$, a cargo de la gestión del territorio metropolitano, han habilitado cinco rellenos sanitarios para recibir los residuos de la ciudad. Uno ha sido habilitado por la municipalidad del Callao, tres por la municipalidad de Lima y uno por la municipalidad de Huarochirí (ubicada en la periferia de la zona urbana). Así, en menos de diez años, la gestión de los residuos ha mejorado grandemente en Lima. Antes de la ley del 2000, los residuos eran recolectados y luego directamente vertidos en botaderos (sin ningún control) por las municipalidades, a falta de infraestructura adecuada para su recepción.

7 La metrópoli de Lima es administrada por dos niveles de municipalidades. Las municipalidades provinciales actúan a escala amplia y existen dos, correspondientes a la zona urbana (las provincias de Lima y Callao), rodeadas por provincias periféricas a la ciudad. Al nivel inferior de estas encontramos los distritos que tienen su propio municipio independiente. La provincia de Lima tiene 43 distritos y el del Callao 6.
En el año 2008, según las estimaciones de las municipalidades de Lima y del Callao, el $86 \%$ de los residuos sólidos terminaban en los cinco rellenos sanitarios habilitados. Lima se ubica así en el promedio de las ciudades latinoamericanas en lo que respecta a las tasas de residuos controlados y correctamente tratados (CEPAL, 2010). El tratamiento de los residuos se hace por enterramiento. $\mathrm{Y}$ aunque numerosos países buscan atenuar la utilización de esta técnica, en Lima el enterramiento permite limitar la propagación de los desperdicios, la infiltración de los lixviados o las emisiones de gas. Se trata, pues, de un avance significativo.

Después de haber evacuado los residuos y limitado el riesgo sanitario de corto plazo, Lima ha podido controlar el enterramiento y los almacenajes de un modo más adecuado. Asimismo, el riesgo ambiental de largo plazo está mejor controlado, aun cuando las dificultades persisten.

\subsection{La importancia de las autoridades locales}

Ese sistema existe en la mayor parte de los distritos de la ciudad de Lima. Los barrios acomodados han sido los primeros en beneficiarse de él, seguidos por los barrios de clase media y ciertos sectores populares, que progresivamente se han consolidado. Los barrios construidos según procesos informales han ido obteniendo poco a poco acceso a las infraestructuras urbanas básicas (agua, electricidad, vialidad, recolección de basura, etc.). Aproximadamente un 50\% de las viviendas de Lima son de origen informal (Calderón, 2009). 
Figura 1. Mapa del manejo de los residuos sólidos en Lima

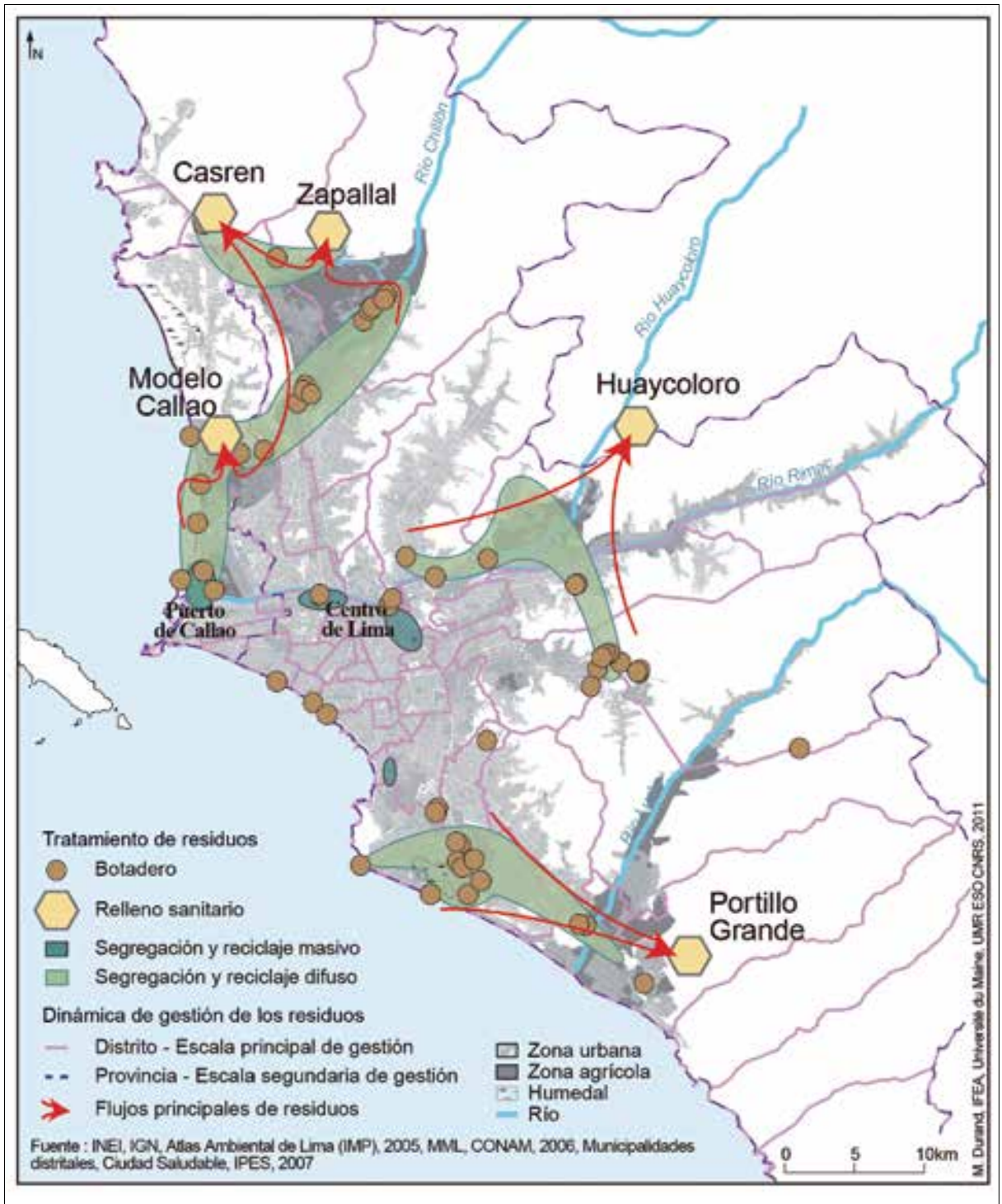

Fuente: INEI, IGN, Atlas Ambiental de Lima (IMP), 2005, MML, CONAM, 2006, Municipalidades distritales, Ciudad Saludable, IPES, 2007. 
Si el $86 \%$ de los residuos sólidos domésticos de Lima terminan hoy en los rellenos sanitarios, es decir, son administrados según el sistema de "gestión pública", es porque la mayor parte de los barrios populares están consolidados. Sin embargo, todavía un gran número de habitantes no tiene acceso a un servicio de gestión de residuos. Además, muchos de los que acceden al servicio no lo reciben en buenas condiciones, pues persisten problemas en la frecuencia de la recolección, en la distancia de las viviendas, en el mal equipamiento de los camiones, etc. Una de las características de Lima-Callao es que se trata de una ciudad muy fragmentada política y administrativamente, ya que existen 49 municipalidades distritales que cubren el territorio metropolitano. La colecta y evacuación de los residuos domésticos está bajo la responsabilidad de los municipios distritales. Solo el tratamiento (la puesta en los rellenos sanitarios) es competencia de las dos municipalidades provinciales, que actúan más eficazmente a escala más amplia.

Ahora bien, existe una gran disparidad de rentas entre los distintos municipios de la ciudad. La renta anual promedio por habitante en el distrito acomodado de San Isidro es de $2.852^{\circ} \mathrm{S}$./ ( soles $^{8}$ ), contra los $100^{\circ} \mathrm{S} . /$ de la de Comas ${ }^{9}$. En consecuencia, el servicio de colecta es más o menos eficaz en función de la jurisdicción. En ese contexto, el objetivo para las autoridades es, frecuentemente,

8 El sol peruano es, según las fechas, más o menos equivalente a 0,33 US\$

9 Datos de la Encuesta RENANU 2008 (Registro Nacional de Municipalidades) del INEI. ofrecer a sus conciudadanos un territorio propio desembarazado de residuos. El destino final de estos es una preocupación de segundo nivel. En otras municipalidades, como veremos, suelen implementarse soluciones diferentes de modo más o menos espontáneo. La presión cada vez más fuerte ejercida por el Estado y las municipalidades provinciales permite avanzar en un mejoramiento de la etapa técnica de la eliminación de los residuos.

\section{La especificidad de los países en desarrollo: el sistema de "autogestión"}

Opuesto al sistema precedente, oficialmente ponderado como el único modelo a seguir por todos los actores públicos, existe un segundo sistema de gestión de los residuos, que se desliza por los intersticios dejados por el sistema de "gestión pública". Este último no es completamente eficiente en ciertos rubros, tales como la recolección de desperdicios en los barrios más pobres (menos accesibles, menos solventes, etc.) o el reciclaje de los residuos. Se habla de un sistema de "autogestión". Los circuitos informales, que funcionan al margen de la ley, se han puesto en marcha para paliar las carencias. Así, paralelamente a la "gestión pública" de los residuos, funciona una red de actores que interviene a diversas escalas en la recolección, selección, reciclado y reventa de los residuos. La figura 2 muestra la organización teórica de esas redes, teniendo en cuenta que cada actor frecuentemente representa varios roles. 
Figura 2. Imbricación de los actores de la gestión de los residuos en Lima.

Los colectores de residuos

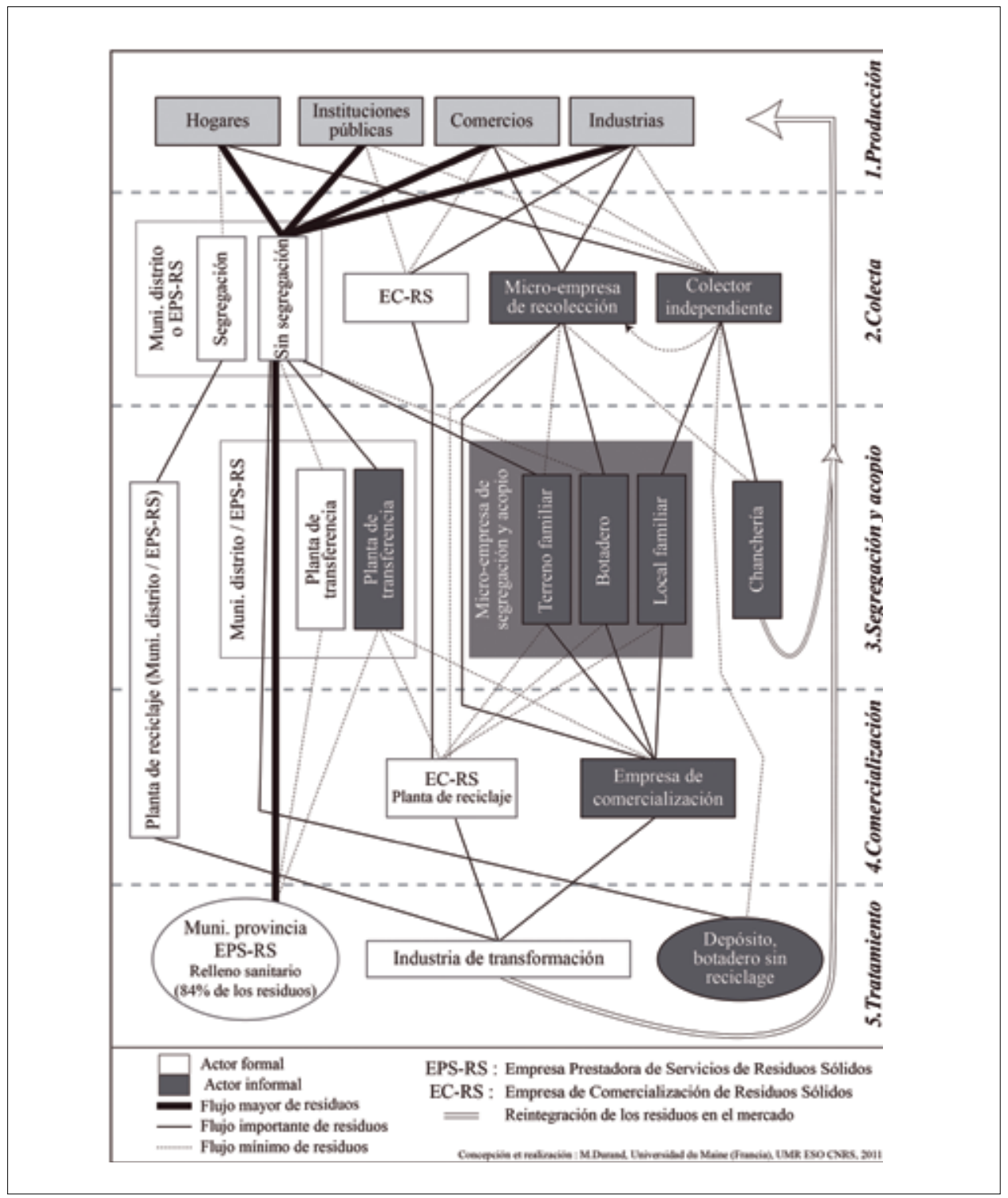

Fuente: M. Durand, Universidad du Maine (Francia), UMR ESO CNRS, 2011 


\subsection{Los colectores de residuos}

Los colectores de los residuos son aquellos que se encargan de recoger directamente en la calle. Esta colecta es evidentemente informal, puesto que los recicladores informales no son autorizados. La obtención de una autorización para el reciclaje es muy estricta en el Perú. Las empresas que se lanzan en esta operación deben responder a exigentes criterios de protección sanitaria y ambiental, que corresponden a criterios de las instituciones internacionales. Solo una parte de los residuos industriales es oficialmente recolectada para ser reciclada.

Los colectores informales trabajan en unos casos solos (colectores independientes) y en otros agrupados en microempresas de recolección. Ellos pueden actuar de dos modos. Primero, interviniendo en barrios donde las municipalidades no participan. Los pequeños colectores se instalan en los barrios para recoger una parte de los residuos. Aunque a veces existen acuerdos tácitos con los pobladores, la mayor parte del tiempo simplemente recogen los residuos reciclables (plásticos, vidrios, metales, etc.) a fin de limpiarlos, segregarlos y luego venderlos. Los desperdicios residuales son frecuentemente quemados o arrojados en los espacios vacíos (eriales, cursos de agua, cunetas, etc.).

El segundo modo de intervención de los recicladores consiste en circular por los barrios de clases medias o acomodadas, donde la colecta municipal es bien efectuada. Se trata de recicladores callejeros, quienes actúan generalmente por la noche a fin de rebuscar los cubos de basura depositados en la vía pública, recogiendo únicamente los residuos reciclables antes del paso de los camiones municipales, procurando recuperar los desperdicios con el mayor valor agregado. Su actividad, aunque informal, es frecuentemente tolerada, no obstante unos distritos son más severos que otros. A menudo, los serenazgos (policía distrital) dejan a los recicladores actuar libremente por las noches, puesto que no perturban la circulación y alteran menos la imagen del vecindario. En los distritos más acomodados, las autoridades intentan hacer desaparecer a los recicladores, percibidos como asociados a la pobreza, a la insalubridad, e incluso, a la peligrosidad.

\subsection{Los segregadores y acopiadores de residuos}

La segunda categoría de recicladores corresponde a unas microempresas siempre informales, aunque más grandes que los colectores: las microempresas de segregación y acopio. Compran a los colectores los residuos reciclables ya preseleccionados. El precio de los residuos plásticos, metálicos, vidrio u otros, es fijado en función de la cotización del mercado. Algunos segregadores compran ocasionalmente sus residuos a los basureros municipales. En la ruta a los rellenos sanitarios, los camiones municipales se detienen en los locales de los microempresarios de segregación y acopio para venderles el fruto de su colecta.

Existen muchos tipos de segregadores, en función de su tamaño y su localización en la ciudad. Una parte de ellos está ubicada en 
los barrios periféricos próximos al centro histórico de Lima, en las principales zonas de mercados y comercio, así como en los barrios modernos. Se trata de barrios que cumplen la función de receptores de residuos, y esto ha sido así desde la fundación de la ciudad. Hoy día estos barrios se han especializado en el acopio temporal de los residuos, antes de expedirlos a la periferia de la ciudad donde serán revendidos a las industrias.

Los recicladores trabajan a veces en un local familiar, que sirve al mismo tiempo de vivienda. La concentración de los recicladores en muy importante pues facilita sus procesos. Ellos realizan una selección muy precisa de los residuos (diferenciando tipos de plásticos, por ejemplo), los limpian y los almacenan, antes de poder venderlos en mayor cantidad a un comerciante mayorista o simplemente a la industria que la transforma en nueva materia prima.

Otros seleccionadores están ubicados en las periferias extremas de la ciudad, en los barrios más recientes y no consolidados, frecuentemente próximos a los ríos. Se trata de empresas que funcionan sobre un amplio terreno familiar (que asimismo sirve de vivienda), lo que les permite almacenar cantidades más grandes de residuos. Generalmente se ubican en el trayecto hacia los rellenos sanitarios, lo que les facilita la interceptación de los residuos en el camino. Por su parte, las microempresas que trabajan en un botadero corresponden a criterios muy similares. Sus labores, sin embargo, se realizan sobre un territorio más grande, con un flujo de residuos aún más importante.
Otros actores del reciclaje se ubican dentro de la misma categoría de selección: los criadores de chanchos. En los barrios populares de Lima, un buen número de habitantes crían chanchos, alimentándolos con desperdicios orgánicos (restos alimentarios) de la población. Esta técnica permite volver a usar una gran parte de los residuos, puesto que los desperdicios orgánicos representan más del $50 \%$ de los residuos domésticos de Lima (Grupo Gea, 2005). Las condiciones de crianza de estos animales y la reutilización de los desperdicios plantean grandes riesgos sanitarios, tanto para los criadores como para los consumidores. Las autoridades locales son más severas con este tipo de reciclaje que, pese a todo, está muy extendido entre las familias más pobres.

\subsection{Comercialización y transformación de los residuos}

Posterior a la colecta y segregación de los residuos, vienen las etapas de la comercialización y la transformación. Se trata del rescate de los residuos por los mayoristas, generalmente especializados en un solo tipo de estos. A veces, las tareas de comercialización las efectúan las microempresas de segregación y acopio, en otros casos se trata de empresas específicamente dedicadas a esta labor. A partir de este escalón son más numerosas las empresas formales, a consecuencia de la mayor dimensión de esta parte del negocio. Las empresas de comercialización, formales o no, revenden sus residuos a unas industrias que efectúan la transformación en "materias primas secundarias". La autorización de 
las empresas legales les permite trabajar únicamente con residuos industriales, pero, en la práctica, la diferenciación con los residuos domésticos es compleja.

Si las prácticas del sistema de "autogestión" de los residuos tienen indudable utilidad para la sociedad urbana limeña, engendran asimismo numerosos riesgos. La salud de los trabajadores y el ambiente local son fuertemente afectados. El impacto ambiental puede, sin embargo, ser discutido en relación con el interés ecológico de la valorización de los residuos efectuada. Vale tomar en cuenta que la problemática ambiental a gran escala se resuelve entonces en detrimento del ambiente local. Finalmente, las condiciones de informalidad llevan a que esta actividad sea reservada a las poblaciones más pobres y marginales.

\section{Aprovechar la realidad compleja de las ciudades en desarrollo, para implementar un sistema de "gestión compartida" de los residuos}

Los dos sistemas anteriormente considerados pueden ser examinados tomando en cuenta dos factores cuya observación permitirá proponer un tercer sistema pertinente a las particularidades de una ciudad en desarrollo. Este sistema se apoya sobre "el compartimiento de las cargas y responsabilidades de manejo entre los residentes y los actores institucionales" (Jaglin, 1995), asociando también todos los actores informales de la gestión de residuos sólidos. La puesta de relieve del sistema de gestión compartida de los residuos está justificada por la convergencia de esos dos factores, y también por un trabajo empírico.

\subsection{Dos factores en juego: "la eficacia de la acción pública" y "la implicación de las poblaciones"}

El primero de esos factores es "la eficacia de la acción pública". La gestión de los residuos será juzgada eficaz cuando permita disminuir - hasta hacer desaparecer- los riesgos sanitarios y ambientales ligados a los residuos. Es decir que los residuos deben ser evacuados al exterior de las zonas habitadas y tratados, a fin de no degradar los recursos naturales necesarios para el funcionamiento de esos mismos espacios urbanos. Después de algunas decenas de años, otra dimensión es añadida a esta eficacia. Se trata de la capacidad de valorizar los residuos, puesto que por mucho tiempo hemos olvidado su valor añadido.

En todos los casos, cuando la población o las autoridades de la ciudad de Lima y las ciudades de América Latina hablan de una gestión eficaz de los residuos, ellos evocan una "gestión pública" eficaz. Basados en los modelos de las instituciones internacionales, el objetivo es que las autoridades se responsabilicen de la gestión de los residuos. El conjunto de esta tarea les es entonces adjudicada, aunque ciertas municipalidades las transfieran a prestatarios privados del servicio.

El segundo factor que entra en juego en la distinción entre los dos sistemas 
observados es la "implicación de la población" en la gestión de los residuos. El sistema de "autogestión" se basa en la participación muy elevada de los hogares (es decir de los productores de residuos). Para que los riesgos asociados a la presencia de residuos disminuyan, la población que los produce debe imperativamente implicarse en los procesos de gestión. Si ellos no hacen el esfuerzo de llevar sus residuos a los recicladores, de quemarlos o de echarlos a los ríos vecinos, los riesgos sanitarios que pesarían sobre ellos serían mucho más graves. Los circuitos informales de gestión de los residuos participan igualmente de esta fuerte implicación de la población en la gestión de los residuos. Se trata esta vez de rivereños creando sus microempresas informales, participando en el bienestar de la comunidad y en el manejo de los residuos. Ellos realizan esta acción con un objetivo lucrativo (hasta si la ganancia es mínima) y lo hacen, no obstante, con detrimento de su salud.

Ahora bien, el modelo hacia el cual quiere dirigirse la mayor parte de las municipalidades de la ciudad, es decir, el sistema de gestión pública, tiene justamente por objetivo suprimir toda implicación de la población. La no implicación en la gestión de los residuos aparece como un factor de confort, marcador de un cierto estatus social, no tocando los residuos y que estos sean evacuados del barrio. En esta visión de la gestión de los residuos, solo las poblaciones más marginalizadas pueden tener contacto directo con esta apetitoso objeto. Por otra parte, el sistema de "gestión pública" quiere tecnificar la operación, permitiendo implicarse solamente a las empresas especializadas.

El hecho de alejar los residuos de la vida cotidiana de los habitantes permite, en cierta medida, limitar los riesgos sanitarios. Ahora bien, es posible hoy en día encontrar puntos intermedios que no asocien sistemáticamente la manipulación de los residuos a ese riesgo. Por otra parte, el alejamiento de los residuos de la vida cotidiana engendra gran desinterés por el devenir de este objeto. Es decir, las poblaciones se preocupan en lo sucesivo en saber si los residuos son bien evacuados de su barrio, y punto. La división político-administrativa muy fuerte de la ciudad de Lima hace que, si el alcalde de uno de los 49 distritos de la ciudad logra hacer evacuar los residuos de su jurisdicción, la población destacará sus méritos, importando poco el destino final de estos ${ }^{10}$. Si el retroceso de la implicación de la población en la gestión de los residuos aporta un cierto confort cotidiano, se hace más difícil tomar en cuenta el conjunto de la cadena de tratamiento y limita la posibilidad del reciclaje, al menos en un primer momento.

Es, pues, la articulación entre estos dos factores lo que permite comprender me-

10 Como para el conjunto de este artículo podemos hacer aquí el paralelo con la gestión de las aguas residuales. La situación es aún más embarazosa en Lima por esas aguas, porque la población tiene acceso a las redes de saneamiento en un rango del $86 \%$ (Censo INEI 2007). Ahora bien, solo el $14 \%$ de las aguas residuales son tratadas. El resto es descargado al mar sin ningún tratamiento. La mayoría de la población de la ciudad, al no tener más contacto con las aguas residuales, no se preocupa de su destino. Las autoridades no buscan entonces ofrecer soluciones para el tratamiento de las aguas residuales (Durand, 2010b). 
jor la imbricación entre los diferentes sistemas teóricos de gestión de residuos. Por otra parte, en un contexto mundial de afirmación de la necesidad de tomar en cuenta la problemática medioambiental, la implementación del reciclaje en la fuente adquiere un nuevo impulso. Ese testimonio, primero circunscrito al discurso de las poblaciones de las ciudades ricas, se arraiga rápidamente en las ciudades en desarrollo. La ecuación que pretende que la preservación del medioambiente sea reservada a los ricos es puesta en tela de juicio en numerosos lugares (Emelianoff, 2010).

En América Latina se asiste al desarrollo de una serie de iniciativas que ponen de nuevo en valor el reciclaje. Ahora bien, esta práctica del reciclaje en la fuente directamente por los hogares, necesita una fuerte implicación de la población. De otra parte, frecuentemente es la falta de implicación de la población lo que hace difícil la implementación de estos sistemas en ciudades del Norte, requiriendo largas y costosas campañas de sensibilización. El distrito acomodado de Surco en Lima ha prolongado su sistema de "gestión pública" de los residuos haciendo una colecta selectiva, lo que lo ha llevado actualmente a las mismas dificultades de desmovilización de las poblaciones en la selección dentro del hogar. Observamos un forzado retorno de la necesidad de la implicación de los diferentes actores - luego de haber logrado erradicarlos con el sistema de "gestión pública" - en la gestión de los residuos, particularmente a aquellos habitantes que los producen.

\subsection{Pasar directamente a un sistema de "gestión compartida"}

Sobre la base de esta observación, el reto de la gestión de los residuos en los países en desarrollo es lograr conjugar la eficacia de las autoridades, apoyándose plenamente en la implicación de la población ya existente. Se trata de conjugar la evolución técnica y la confianza en los actores locales.

En la situación actual, las municipalidades buscan llevar a cabo una acción eficaz, apoyándose en los indicadores de la gestión de los residuos proporcionados por las instituciones internacionales. Ahora bien, este objetivo es imposible de alcanzar a medio plazo, puesto que las finanzas públicas no son las mismas que en otros países. De otra parte, las ciudades del Norte actualmente buscan reanudar con la práctica del reciclaje y la manipulación cotidiana de los residuos por la población. ¿Por qué, pues, intentar hacer desaparecer esta participación en países en desarrollo, cuando se la intenta restituir en otras partes? Finalmente, la lucha encarnizada contra las prácticas informales de los recicladores a corto plazo es vana, en un contexto urbano donde gran parte de la población vive en condiciones de gran pobreza y la informalidad representa más de la mitad de las actividades económicas (Barragán, 2005).

En consecuencia, las ciudades en desarrollo pueden sacar ventaja de aquellos elementos percibidos como sus debilidades, pasando directamente de una "autogestión" a una "gestión compartida", sin pasar necesariamente por el sistema de 
Figura 3. Evolución de los sistemas de gestión de los residuos en las ciudades en desarrollo.

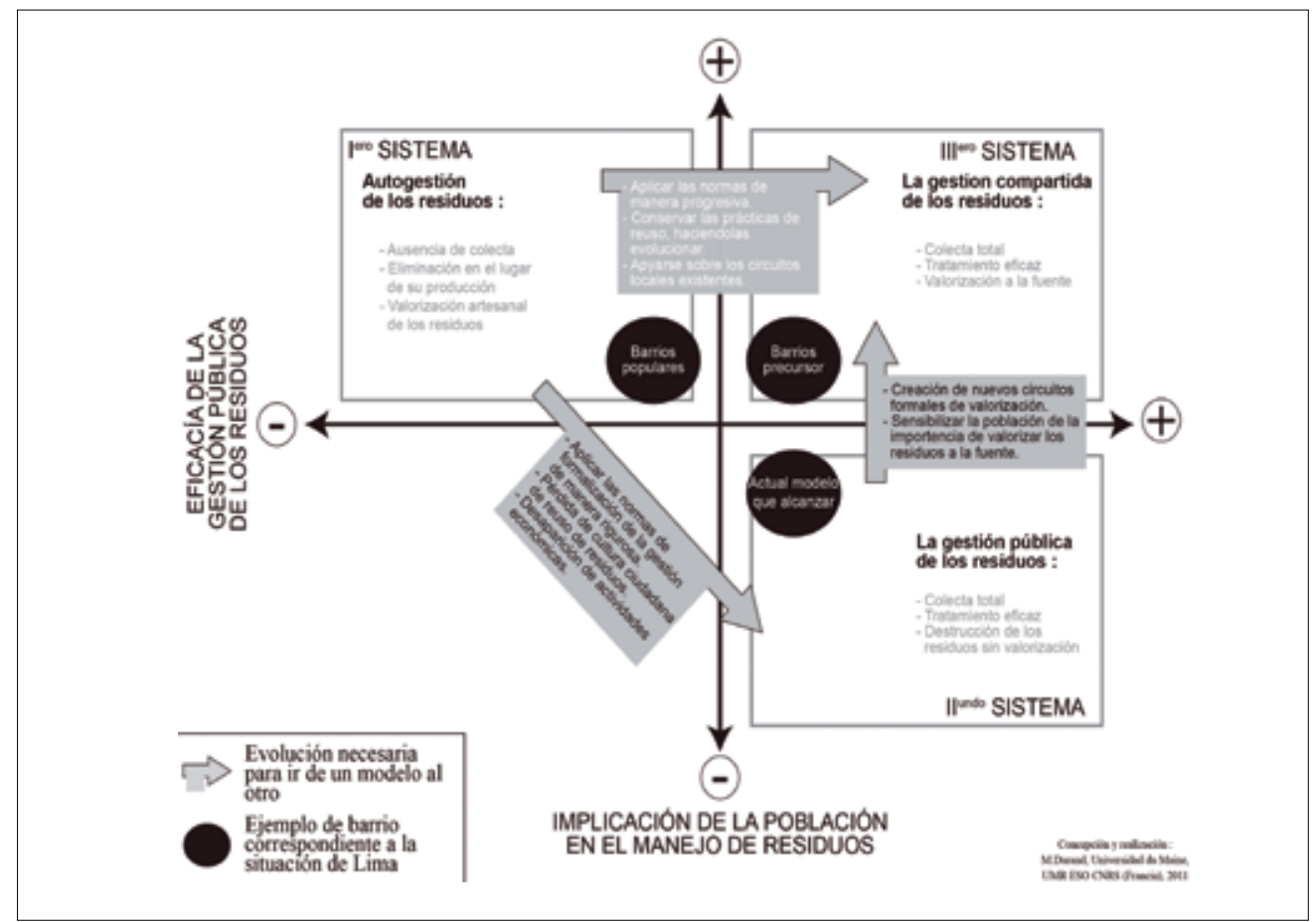

Fuente: M. Durand, Universidad du Maine (Francia), UMR ESO CNRS, (Francia), 2011

“gestión pública”. El paso del primero al segundo y finalmente al tercer sistema necesita de una serie de adaptaciones largas y costosas de implementar. Para pasar de un sistema de "autogestión" a uno de "gestión pública", es necesaria una inversión financiera importante para modernizar las infraestructuras. Este esfuerzo en gran parte ya ha sido realizado en Lima con la puesta en funcionamiento de los cinco "rellenos sanitarios" entre el 2000 y el 2010 (Alternativa, 1999). El paso de un sistema a otro, sin embargo, se hace perdiendo un activo importante: la implicación de la población.
Ahora bien, esta implicación de la población es indispensable para la implementación de un sistema de gestión compartida. Al seguir las recientes evoluciones de las ciudades desarrolladas, este sistema busca reciclar la mayor parte de los residuos domésticos, efectuando una colecta selectiva directamente en la fuente. Esto exige tareas compartidas entre las autoridades, que aseguren la colecta, el tratamiento y la valorización de los residuos, y la población tiene que encargarse de la selección preliminar. Esta implicación de la población existe aún en los sistemas de autogestión, que 
están siendo progresivamente erradicados por los sistemas de gestión pública. La apuesta del pasaje al tercer sistema - de gestión compartida - es la de conservar el mejoramiento técnico salido del sistema de gestión pública, rescatando al mismo tiempo el factor de implicación de la población proveniente del sistema de autogestión.

Las ciudades de los países en desarrollo tienen mucho interés en conducir al mismo tiempo la modernización de las infraestructuras y el desarrollo de una cultura del reciclaje, a fin de pasar directamente del sistema de autogestión al sistema de gestión compartida. Este pasaje permitiría enfocar directamente las inversiones financieras hacia las infraestructuras que faciliten el reciclaje de los residuos. De este modo se obtendría provecho de una competencia ya existente en la manipulación de residuos por parte de la población. Sería posible, igualmente, apoyarse sobre la red intermediaria de recicladores, a fin de perfeccionar su gestión de los residuos y mejorar sus condiciones de trabajo. Evidentemente es necesario que los recicladores evolucionen en las condiciones de manipulación de los residuos para evitar la propagación de riesgos. El pasaje directo a un tercer sistema sería entonces facilitado por el hecho de que no existe todavía desdén por el objeto "residuos". El respaldo a los circuitos (informales) de recicladores sería entonces un activo para las autoridades locales. Esta es, de otra parte, la óptica con la que ya trabaja un cierto número de municipalidades en Lima.

\subsection{Las experiencias de "gestión compartida" de residuos en Lima}

La reflexión expuesta antes se apoya en el hecho de que un cierto número de municipalidades limeñas ha comenzado a implementar estos sistemas de gestión compartida de residuos. La municipalidad de Surco, uno de los distritos de Lima, ha organizado una recolección selectiva y el reciclaje desde el año 2000. Surco es, sin embargo, uno de los distritos más ricos de la ciudad. Se trata de la reproducción exacta de los sistemas de reciclaje implementado en las ciudades del Norte.

Otros distritos han imaginado mecanismos más originales, trabajando directamente con los recicladores informales de las calles, a fin de mejorar la recolecta de una parte, la tasa de residuos reciclados de otra parte, y finalmente las condiciones de trabajo de los recicladores. Se trata, por ejemplo de los distritos de Villa El Salvador, Los Olivos y el Cercado del Callao. En cada uno de estos casos, los habitantes son incitados a entregar sus residuos, previamente seleccionados y separados en bolsas plásticas de colores a unos recicladores autorizados por la municipalidad. El interés de los habitantes es directo, pues a cambio de una bolsa de residuos reciclables, el reciclador entrega un bono al vecino, permitiéndole beneficiarse de una reducción sobre su próximo impuesto a la recolección de basura doméstica. Los recicladores se encargan así mismo de la sensibilización 
de la población respecto a la importancia del reciclaje y sus técnicas. En una primera etapa, esta sensibilización se hace con el apoyo de educadores municipales

En esta operación, la alcaldía completa el servicio. Los recicladores, en efecto, realizan una parte del servicio de colecta de residuos, facilitando el trabajo de los basureros municipales. Para los recicladores informales esta colaboración tiene mucho interés: ante todo les ofrece mayor estabilidad, no siendo ya perseguidos por la policía, y tienen además la responsabilidad de un cierto número de manzanas, evitando así la competencia con otros recicladores. Por otra parte, la municipalidad los equipa con guantes, máscaras, chalecos de identificación y carretillas, mejorando sus condiciones de trabajo y limitando los riesgos sanitarios a los que se exponen. En la mayor parte de los casos, los recicladores permanecen como propietarios de los residuos que han recolectado. Ellos lo revenderán entonces a las empresas de comercialización y reciclaje. Si estas empresas son informales, la municipalidad intenta progresivamente formalizar la relación, favoreciendo a las empresas legales, estimulándolas a respetar las condiciones sanitarias y sociales del trabajo, así como a proteger el medioambiente local. En otros casos, como en el distrito de Los Olivos, la municipalidad intenta asegurar la recompra y la comercialización de los residuos reciclables

\section{Conclusión: cuando el "sistema compuesto" permite mutualizar los esfuerzos para manejar los residuos}

La implementación progresiva del sistema de "gestión compartida" ocasiona, sin embargo, un límite mayor, pues obliga a las autoridades locales a ponerse en una situación de ilegalidad. Es decir que las municipalidades deben colaborar con unos recicladores que no tienen autorización para ejercer esta actividad debido a las malas condiciones sanitarias y sociales en las cuales trabajan.

A pesar de eso, el sistema de gestión compartida implementado en ciertos distritos de Lima posibilita ir a un mejoramiento progresivo a tres niveles: las condiciones de trabajo de los recicladores, la calidad del servicio ofrecido a la población y la protección del ambiente. Permite asimismo salir de la hipocresía anterior consistente en creer que es posible alcanzar unas normas de calidad bastante elevadas - como las practicadas en los países desarrollados en un contexto de debilidad presupuestaria e institucional de las autoridades. Estas normas tienen el mérito de fijar los objetivos por alcanzar a largo plazo. Su aplicación es, no obstante, irrealista a corto y medio plazo. El pasaje por el sistema compartido así, permite aproximarse a las normas en vigor, mantenerlas en la mira a fin de mejorar progresivamente - probablemente en el curso de varios decenios- el sistema de gestión de los residuos en las ciudades en desarrollo. 
Se trata así de mutualizar los esfuerzos de las autoridades, de los empresarios privados informales (pero también de los formales) y de la población. El objetivo no es sustituir un sistema formal por uno informal, sino de mejorar la articulación entre estos diferentes sistemas. Esta práctica existe para la gestión de los residuos, pero igualmente en otros servicios urbanos, tales como el agua potable, el saneamiento y la electricidad. Se trata del desarrollo de sistemas compuestos, puestos en evidencia por Sylvy Jaglin (2006), en los cuales se yuxtaponen la gestión pública, la autogestión y la gestión compartida. En función de las características de cada distrito, una u otra solución será privilegiada. El conjunto se articula en un sistema compuesto a escala de la ciudad, adaptándose a cada distrito en función de sus potencialidades. Para poder funcionar, los diferentes actores de las ciudades en desarrollo mutualizan sus medios, de modo frecuentemente espontáneo, a fin de hacer funcionar el sistema urbano. El reconocimiento de este sistema por las autoridades locales puede animarlos a intentar organizar mejor esta mutualización.

El estudio de un territorio específico como la ciudad de Lima, permite ofrecer una cartografía (Durand, 2010a) de la repartición espacial entre los diferentes sistemas evocados anteriormente. Es posible así dar luces sobre aquellas lógicas urbanas y sociales más amplias que la sola gestión de los residuos. Esta realidad de la articulación entre los diferentes sistemas de gestión de residuos, facilita el funcionamiento del sistema urbano. Ella expone, sin embargo, a una parte de la población a riesgos importantes. El trabajo efectuado por gestores de residuos y por la ciudad, tomando en cuenta la articulación de los diferentes subsistemas, forma el sistema compuesto que posibilita ir hacia un mejoramiento progresivo, lento pero real y conforme a la situación. De este modo, la metrópoli de Lima logra administrar casi 7.000 toneladas de residuos sólidos por día. 


\section{Literatura citada}

Alternativa (1999). Hacia el plan maestro para la gestión y el manejo de los residuos sólidos en el cono norte de Lima. Lima, Perú: ONG Alternativa, Union Européenne, 79p.

Barles, S. (2005). L'invention des déchets urbains, France: 1790-1970. Paris, France: Champ vallon, $297 \mathrm{p}$.

Barragán, P. (2005). Magnitud de la economía informal en el Perú y el mundo. Revista gestión en el tercer milenio, 7 (14), 43-51. Lima, Perú: UNMSM.

Barraque, B. (1998). Les services publics d'eau et d'assainissement face au développement durable. Annales des Ponts et chaussées, (87). (9p.). Paris, France : ENPC.

Calderón, J. (2009). La producción de la ciudad formal e informal. En Foro urbano, Los nuevos rostros de la ciudad de Lima (p.17-30). Lima, Perú: Colegio de Sociólogos del Perú.

Carre, M. (2010). Déchets et conflits métropolitains : les territoires de la gouvernance environnementale à Buenos Aires. Revue RITA, (4), 12p.

CEPAL (2010). Anuario estadístico de América Latina y el Caribe. Santiago de Chile: Nations Unies, 310p.

Durand, M. (2010a). Gestion des déchets et inégalités environnementales et écologiques à Lima (Pérou) : entre vulnérabilité et durabilité. Thèse de doctorat. Université de Rennes 2, Rennes, France. 458p.

Durand M. (2010b). Inégalités écologiques et eaux usées à Lima. En G. Schneier-Madanes. L'eau mondialisée (p.439-454.). Paris, France: La Découverte.

Dureau, Goueset \& Mesclier (2006). Géographie de l'Amérique Latine. Rennes, France: Presses Universitaires de Rennes, 361p.

Emelianoff, C. (2010). Durabilité urbaine, modes de vie et solidarités à long rayon d'action. En O. Coutard \& J-P. Levy. Ecologies urbaines (p.302-327.). Paris, France: Economica.

Fernández, L. (2012). Dynamique du recyclage spontané, regard croisé sur les villes de Montevideo et Paris. Actes du séminaire Mise en valeur des déchets, processus et enjeux. Réseau Sociétés Urbaines et Déchets MSH de Tours, Tours, France, 6 février 2012, 38p.

Grupo Gea (2005). Estudio de composición física de los residuos sólidos domésticos en Lima. Lima, Perú: MML, 55p. 
Jaglin, S. (1995). Gestion urbaine partagée à Ouagadougou, pouvoirs et périphéries 19831991. Paris, France: Karthala, 659p.

Jaglin, S. (2006). Le défi de la desserte en eau des quartiers pauvres en Afrique subsaharienne: quel(s) service(s) et avec quelles conséquences sur la cohésion urbaine? Actes du colloque AFD du 15 juin 2006 Les PPP : de l'eau pour les plus démunis ? (9 p.). Paris, France: AFD.

Lebris, É. (2002). Inscrire la question de l'assainissement et des déchets dans une problématique urbaine. Programme Gestion durable des déchets et de l'assainissement urbains. Paris, France: PSEAU, PDM, 17p.

Oliveira, A. (2008). As alternativas para o gerenciamento dos resíduos sólidos em Curitiba, Contribuição com a melhoria da qualidade. Revue Caminhos de geografia, 9 (27), 116-127. Uberlândia, Brazil: Universidade Federal de Uberlândia.

Tchuikoua, L. (2010). Gestion des déchets solides ménagers à Douala au Cameroun: opportunité ou menace pour l'environnement et la population? Thèse de doctorat. Université de Bordeaux 3, Bordeaux, France, 479p.

Recepción: 20 de diciembre de 2011

Evaluación: 7 de mayo de 2012

Aprobación: 12 de junio de 2012 